\title{
Strong Similarities between the Local Electronic Structure of Insulating Iron Pnictide and Lightly Doped Cuprate
}

\author{
Cun Ye, ${ }^{1}$ Wei Ruan, ${ }^{1}$ Peng Cai, ${ }^{1}$ Xintong Li, ${ }^{1}$ Aifeng Wang, ${ }^{2}$ Xianhui Chen, ${ }^{2}$ and Yayu Wang ${ }^{1,3, *}$ \\ ${ }^{1}$ Department of Physics, State Key Laboratory of Low Dimensional Quantum Physics, \\ Tsinghua University, Beijing 100084, People's Republic of China \\ ${ }^{2}$ Hefei National Laboratory for Physical Science at Microscale and Department of Physics, \\ University of Science and Technology of China, Hefei, Anhui 230026, People's Republic of China \\ ${ }^{3}$ Collaborative Innovation Center of Quantum Matter, Beijing 100084, People's Republic of China
}

(Received 8 April 2014; revised manuscript received 25 January 2015; published 29 April 2015)

\begin{abstract}
One of the major puzzles regarding unconventional superconductivity is how some of the most interesting superconductors are related to an insulating phase that lies in close proximity. Here, we report scanning tunneling microscopy studies of the local electronic structure of $\mathrm{Cu}$-doped $\mathrm{NaFeAs}$ across the superconductor-to-insulator transition. We find that in the highly insulating regime, the electronic spectrum develops an energy gap with diminishing density of states at the Fermi level. The overall line shape and strong spatial variations of the spectra are strikingly similar to those of lightly doped cuprates close to the parent Mott insulator. We propose that the suppression of the itinerant electron states and the strong impurity potential induced by $\mathrm{Cu}$ dopants lead to this insulating iron pnictide.
\end{abstract}

DOI: 10.1103/PhysRevX.5.021013

A key task in unraveling the mystery of unconventional superconductivity is to disentangle the various phases that lie in close proximity to the superconducting (SC) phase. One of the most surprising discoveries in this regard is that the cuprate superconductors, which possess the highest transition temperature $\left(T_{C}\right)$, have an insulating parent compound. How the high- $T_{C}$ superconductivity emerges from doping charges into a Mott insulator has since become one of the greatest challenges in modern condensed-matter physics [1]. Although the answer remains controversial, there is general consensus that the Mott insulator represents a valid starting point for tackling the problem. The recently discovered iron-based superconductors, on the contrary, have a metallic parent state with a well-defined Fermi surface $[2,3]$. This apparently implies a totally different viewpoint for understanding the mechanism of superconductivity. Most theoretical proposals start from the itinerant electron picture and search for the excitations that mediate the Cooper pairs [4,5]. The electron correlation effect, which plays a dominant role in the cuprates, is generally considered to be unimportant in the iron pnictides.

An intriguing question inspired by this sharp contrast is whether there also exists an insulating phase in the iron

\footnotetext{
*yayuwang@tsinghua.edu.cn
}

Published by the American Physical Society under the terms of the Creative Commons Attribution 3.0 License. Further distribution of this work must maintain attribution to the author(s) and the published article's title, journal citation, and DOI.
Subject Areas: Condensed Matter Physics, Strongly Correlated Materials, Superconductivity

pnictides where the electron correlation effect is essential. If the answer is yes, then this insulating phase may be the missing piece of the puzzle that links the pnictides to the cuprates. However, in most iron pnictides, the effect of transition-metal doping is to introduce charge carriers, suppress the spin-density wave (SDW) order, and induce superconductivity $[6,7]$. Overdoping usually leads to a nonSC phase with better metallicity, and an insulating phase is very rare. Interestingly, it was found recently that $\mathrm{Cu}$-doped $\mathrm{NaFeAs}$ exhibits a superconductor-to-insulator transition (SIT) with increasing $\mathrm{Cu}$ content [8]. This system thus provides a unique opportunity to explore the insulating regime of iron pnictides that was not available before. Understanding the electronic structure of the insulating ground state could shed new light on the mechanism of iron-based superconductivity and its connection to the cuprates.

In this work, we use scanning tunneling microscopy (STM) to investigate the local electronic structure of $\mathrm{NaFe}_{1-x} \mathrm{Cu}_{x}$ As with varied $\mathrm{Cu}$ contents across the SIT. We find that $\mathrm{Cu}$ dopants induce a systematic depletion of the itinerant electron spectral weight. In the highly insulating regime, the electronic spectra develop an energy gap with diminishing density of states (DOS) at the Fermi level $\left(E_{F}\right)$, strikingly similar to that in lightly doped cuprates close to the parent Mott insulator. We propose that the suppression of the itinerant electron states and the strong impurity potential induced by $\mathrm{Cu}$ dopants lead to the insulating phase, in which the electron correlation effect might be relevant.

The 111-type NaFeAs is ideal for STM studies due to the charge-neutral cleavage surface between two neighboring 
(a)

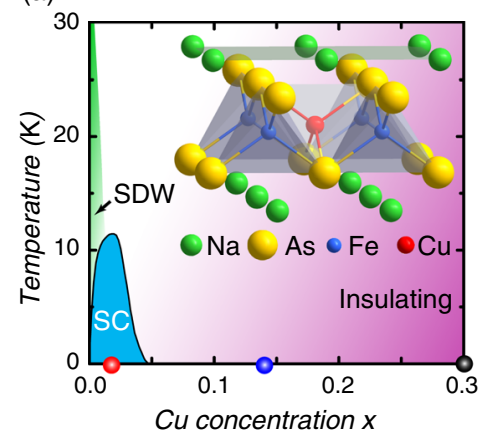

(b)

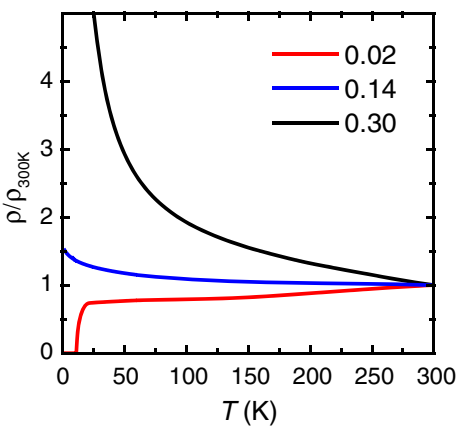

(c)

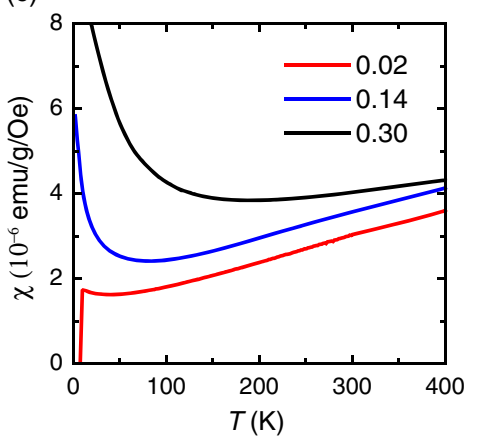

FIG. 1. (a) Schematic phase diagram of $\mathrm{NaFe}_{1-x} \mathrm{Cu}_{x}$ As. Inset: The crystal structure of $\mathrm{NaFe}_{1-x} \mathrm{Cu}_{x} \mathrm{As}$. The gray plane on the top corresponds to the exposed $\mathrm{Na}$ surface layer. (b),(c) Temperature dependence of the in-plane resistivity normalized by the room temperature value and the magnetic susceptibility for the three $\mathrm{NaFe}_{1-x} \mathrm{Cu}_{x}$ As samples.

$\mathrm{Na}$ layers, on which the electronic structure reflects the intrinsic property of the bulk [9]. The inset of Fig. 1(a) depicts the crystal structure of $\mathrm{NaFeAs}$ doped with $\mathrm{Cu}$ impurity. $\mathrm{Cu}$ substitution of $\mathrm{Fe}$ is expected to create a rectangular-shaped pattern on the surface $\mathrm{Na}$ layer, as marked by the gray plane. The main panel of Fig. 1(a) illustrates the schematic phase diagram of $\mathrm{NaFe}_{1-x} \mathrm{Cu}_{x} \mathrm{As}$, which has a substantially suppressed SC dome compared to the widely studied Co-doped NaFeAs. A more fundamental difference is that at the highly $\mathrm{Cu}$-doped regime an insulating phase emerges, whereas increasing Co content makes the system more metallic [10]. The three solid symbols along the $x$ axis in Fig. 1(a) indicate the three doping levels covered in this work, and Fig. 1(b) displays their normalized resistivity $(\rho)$ versus temperature $(T)$ curves. The $x=0.02$ sample is optimally doped with $T_{C}=11 \mathrm{~K}$, but the $x=0.14$ sample shows weak insulating behavior at low $T$. The $x=0.30$ sample represents the highest $\mathrm{Cu}$ doping available so far and shows strongly insulating behavior. Figure 1(c) shows the $T$ dependence of magnetic susceptibility up to $400 \mathrm{~K}$. The general trend is a deviation from the high- $T$ linear behavior [11] with increasing $\mathrm{Cu}$ content, and at $x=0.30$ a pronounced Curie-Weiss-like upturn appears below $T \sim 200 \mathrm{~K}$.

We first use STM to examine the optimally doped sample with dilute $\mathrm{Cu}$ dopants. Shown in Fig. 2(a) is a topographic image acquired on a cleaved surface of the $x=0.02$ sample, where the square lattice with lattice constant $a=4 \AA$ is clearly resolved. The $\mathrm{Cu}$ dopants can be identified as the bright dumbbells centered on the Fe sites, as marked by the dashed boxes, which is consistent with the expected rectangle shown in the inset of Fig. 1(a). Figure 2(b) displays the spatially averaged differential conductance $(d I / d V)$ taken at locations void of impurities. At $T=5 \mathrm{~K}$, the spectrum shows a well-defined SC gap with symmetric coherence peaks on both sides of $E_{F}$. The gap amplitude estimated from the separation between the two peaks is $2 \Delta=9 \mathrm{meV}$, comparable to the $11 \mathrm{meV}$ gap in optimally doped $\mathrm{NaFe}_{1-x} \mathrm{Co}_{x}$ As [12], with $T_{C}=20 \mathrm{~K}$.
With increasing $T$, the gap gradually fills up and the sharp coherence peaks disappear above $T_{C}=11 \mathrm{~K}$.

To scrutinize how the $\mathrm{Cu}$ dopant affects the superconductivity, we show in Fig. 2(c) a series of $d I / d V$ spectra taken in the vicinity of a $\mathrm{Cu}$ dopant (inset). As we (a)

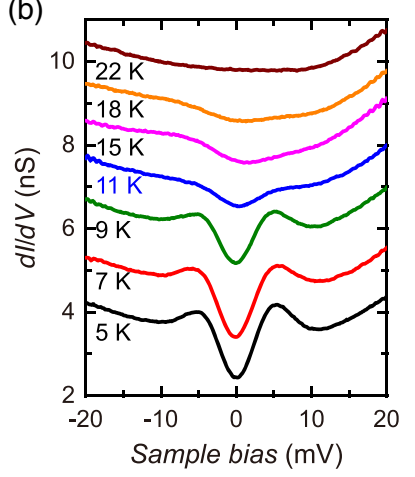

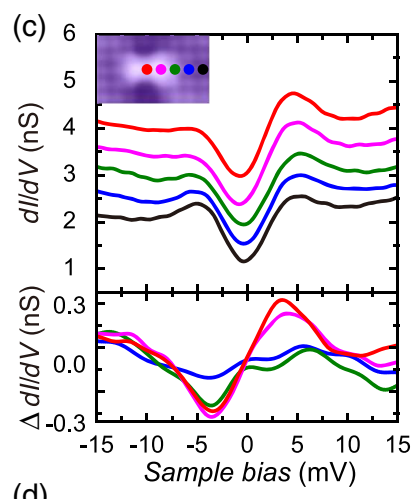

(d)

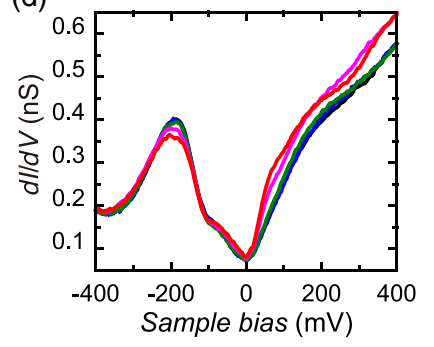

FIG. 2. (a) STM topography on the $x=0.02$ SC sample acquired with bias voltage $V=10 \mathrm{mV}$ and tunneling current $I=300 \mathrm{pA}$. (b) Variable temperature $d I / d V$ spectra (vertically offset for clarity) collected from a defect-free area across $T_{C}=11 \mathrm{~K}$, set up with $V=-22 \mathrm{mV}$ and $I=100 \mathrm{pA}$. (c) Upper panel: $d I / d V$ curves (vertically shifted) at $T=5 \mathrm{~K}$ taken at various locations (labeled by corresponding colored dot in the inset) in the vicinity of an isolated $\mathrm{Cu}$ dopant. The set-up conditions are $V=-50 \mathrm{mV}$ and $I=100 \mathrm{pA}$. Lower panel: Spectroscopic variation around the $\mathrm{Cu}$ dopant, obtained by subtracting the $d I / d V$ curves in (c) with the one taken 4 lattice away (black). The colors are the same as in (c). (d) Large bias $d I / d V$ spectra taken at the same spots as in (c) with $V=-500 \mathrm{mV}$ and $I=100 \mathrm{pA}$. 
move towards the impurity center, the main effect is the suppression (enhancement) of the coherence peak on the negative (positive) bias side, whereas the gap amplitude remains the same. These features are similar to those reported on Cu-doped $\mathrm{NaFe}_{1-x} \mathrm{Co}_{x} \mathrm{As}$ [12]. Figure 2(d) shows the $d I / d V$ curves taken on the same locations but over a larger bias range. The overall line shape is similar to that in Co-doped $\mathrm{NaFeAs}$, showing a peak at negative bias around $-200 \mathrm{mV}$ and a DOS minimum right at $E_{F}$. The most prominent effect of the $\mathrm{Cu}$ dopant here is to cause a suppression of the $-200 \mathrm{mV}$ peak, especially for the two spots close to the impurity site. Such local spectral weight suppression is absent in the Co-doped case.

We next move to the weakly insulating $x=0.14$ sample. The topography shown in Fig. 3(a) becomes much more disordered because of the higher level of $\mathrm{Cu}$ doping, making it difficult to resolve the atomic lattice. Figure 3(b) displays nine representative $d I / d V$ curves measured on various locations marked by the colored dots. The $-200 \mathrm{mV}$ peak is continuously suppressed, presumably due to increased $\mathrm{Cu}$ content if we assume that the effect of the $\mathrm{Cu}$ dopant is the same as that shown in Fig. 2(d). On the positive bias side, however, the unoccupied states are almost identical across the whole sample. Shown in Fig. 3(c) are low-bias $d I / d V$ curves measured on the same locations, which reveal a peak near $-20 \mathrm{mV}$ and a dip around $E_{F}$. More interestingly, the low-energy electronic state is closely correlated with the height of the $-200 \mathrm{mV}$ peak. The spatial distribution of the DOS can be directly visualized by $d I / d V$ maps taken at varied biases. As shown in Fig. 4(a), the $d I / d V$ map taken at $V=-200 \mathrm{mV}$ exhibits strong spatial separation into patches with typical size around $2 \mathrm{~nm}$. The $d I / d V$ map taken at $V=-15 \mathrm{mV}$ [Fig. 4(b)] shows similar spatial variations and positive correlation with that in Fig. 4(a). Therefore, the peak-dip feature near $E_{F}$ is more pronounced in areas with a large DOS peak around $-200 \mathrm{mV}$. In contrast, the $d I / d V$ map taken at $V=+200 \mathrm{mV}$ [Fig. 4(c)] is much more homogeneous,

(a)

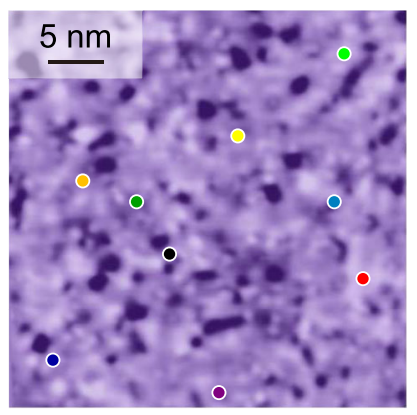

(b)

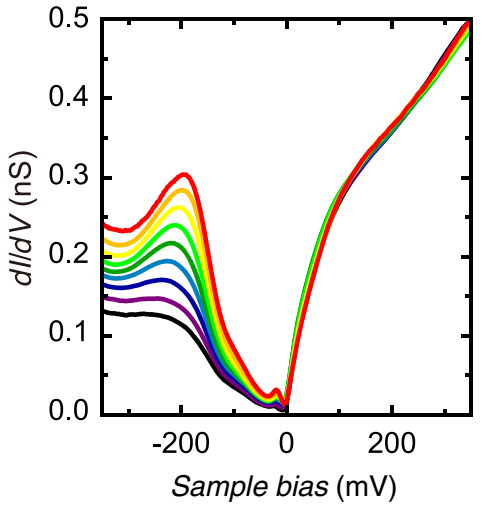

indicating the insensitivity of unoccupied states to $\mathrm{Cu}$ doping.

Finally, we reach the strongly insulating $x=0.30$ sample. As shown in Fig. 5(a), the topography becomes even more disordered with very small granular size down to $1 \mathrm{~nm}$ scale. The large bias $d I / d V$ curves shown in Fig. 5(b) exhibit a further suppression of the occupied state spectral weight compared to that of the $x=0.14$ sample. More interestingly, a closer examination of the low-energy electronic state reveals some fundamentally new features. Figure 5(c) shows that the spectra around $E_{F}$ develop a small energy gap, and for some of the curves (e.g., the black one), the DOS at $E_{F}$ is truly zero. The vanishing Fermi surface in this heavily doped regime is apparently consistent with the strongly insulating resistivity behavior. Moreover, there is also a close correlation between the high-energy and low-energy electronic states. The areas with strongly suppressed occupied state spectra, presumably with higher $\mathrm{Cu}$ content, possess a larger gap size (up to $20 \mathrm{meV}$ ) and smaller residual DOS at $E_{F}$. These features can be directly visualized by the $d I / d V$ maps measured at varied bias voltages. As shown in Figs. 6(a) and 6(b), the locations with strongly suppressed DOS at $-200 \mathrm{mV}$ have much reduced residual DOS at $E_{F}$, as well as a larger energy gap as illustrated by the gap map displayed in Fig. 6(c). The correlation relations between the various quantities are summarized in Fig. 6(d).

A more remarkable feature can be revealed by comparing the spectra of the $x=0.30 \mathrm{NaFe}_{1-x} \mathrm{Cu}_{x}$ As with those of lightly doped cuprates close to the Mott insulator limit. Figure 5(d) shows the $d I / d V$ curves taken on various locations of Na-doped $\mathrm{Ca}_{2} \mathrm{CuO}_{2} \mathrm{Cl}_{2}$ with small hole concentrations [13]. The overall spectral patterns of the insulating iron pnictide and lightly doped cuprate are nearly mirror symmetric when they are put side by side. Over a large bias range, both systems exhibit strongly asymmetric spectral line shape, where one side shows a steep increase and the other side with lower spectral weight

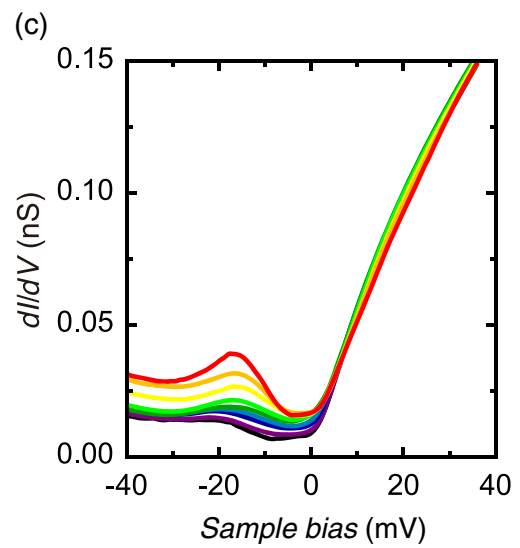

FIG. 3. (a) STM topography on the $x=0.14$ sample acquired with $V=-50 \mathrm{mV}$ and $I=40 \mathrm{pA}$. (b) $d I / d V$ curves taken at locations marked by the colored dots in (a) with set-up conditions $V=400 \mathrm{mV}$ and $I=180 \mathrm{pA}$. A close-up look into energy range around $E_{F}$ is displayed in (c). 
(a)

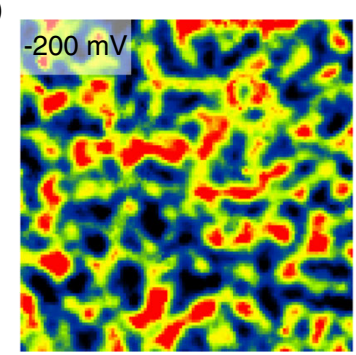

$0.12 \mathrm{nS} \quad 0.30 \mathrm{nS}$

(c)

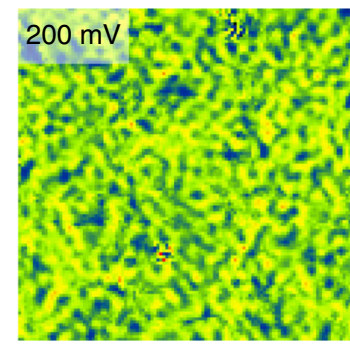

$0.27 \mathrm{nS} \quad 0.35 \mathrm{nS}$ (b)
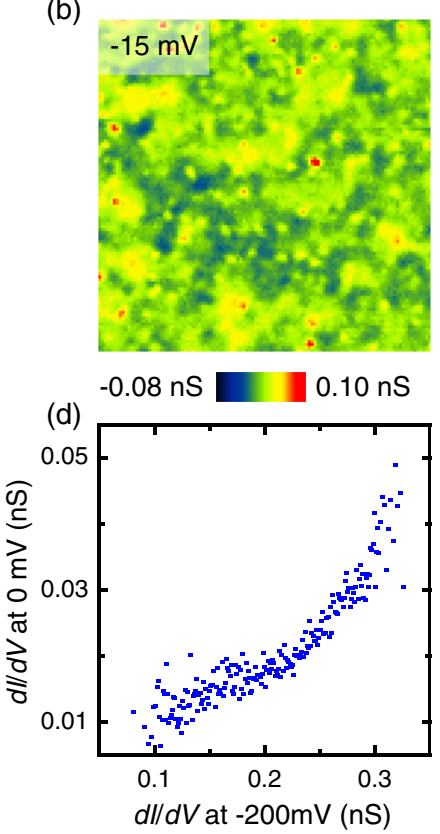

FIG. 4. (a)-(c) $d I / d V$ maps obtained in the same field of view as in Fig. 3(a) with bias voltages $-200,-15$, and $200 \mathrm{mV}$ respectively. (d) Cross-correlation plot of the $-200 \mathrm{mV}$ (a) and $-15 \mathrm{mV}$ (b) $d I / d V$ maps, demonstrating a clear positive correlation.

shows a peak. At low bias, both systems develop a small energy gap with the bottom pinned right at $E_{F}$. In both systems, the size of the gap and the residual DOS at $E_{F}$ show systematic evolution with the local doping level as manifested by the large bias spectra.

The local electronic structure of insulating $\mathrm{NaFe}_{1-x} \mathrm{Cu}_{x} \mathrm{As}$ and its striking similarity to lightly doped cuprates are remarkable features that have never been observed before in any iron pnictides. The key issue here is the origin of the insulating phase, and the first question to tackle is the effect of $\mathrm{Cu}$ doping on the electronic structure of $\mathrm{NaFeAs}$. This is a highly nontrivial task given the fact that, for most iron pnictides, the low-energy electronic structure originates from five partially filled $\mathrm{Fe} 3 d$ orbitals lying near $E_{F}$. Some of the orbitals are itinerant, and hence, are responsible for the Fermi surface formation and metallicity [2]. The other orbitals are more localized, and provide the source for local magnetic moments and spin dynamics [3]. The coexistence and coupling between the local moments and itinerant electrons are responsible for much of the complexities and rich electronic states in the iron pnictides [14-16].

Although there are still controversies regarding the charge and spin state of the $\mathrm{Cu}$ dopant in iron pnictides [17-20], our $d I / d V$ spectroscopy clearly reveals that the main effect of $\mathrm{Cu}$ doping is to cause a suppression of the electron DOS on the occupied side, especially near $-200 \mathrm{meV}$. Similar spectral (a)

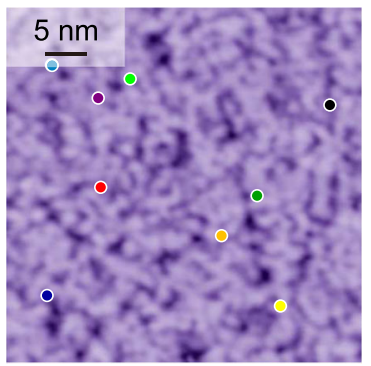

(c)

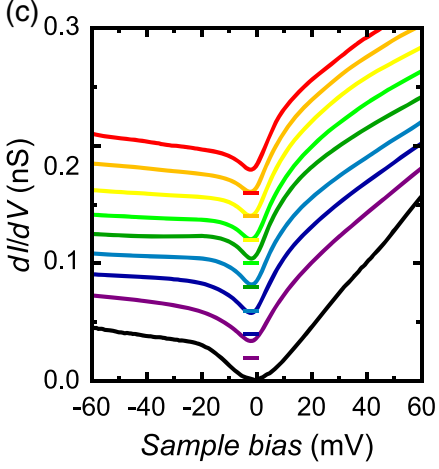

(b)

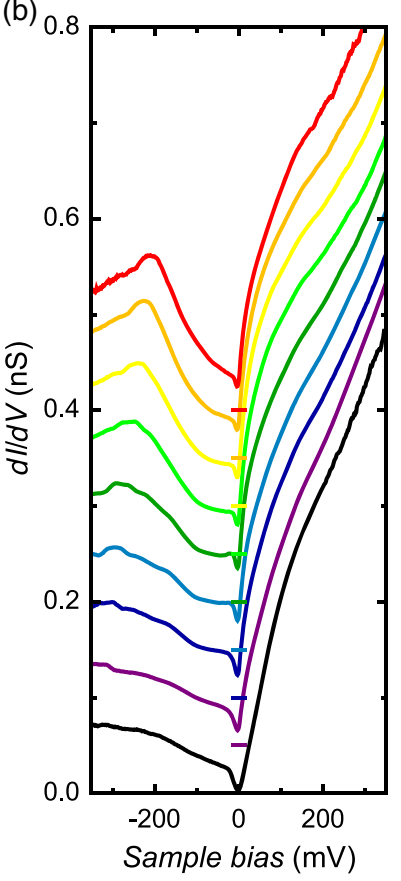

(d)

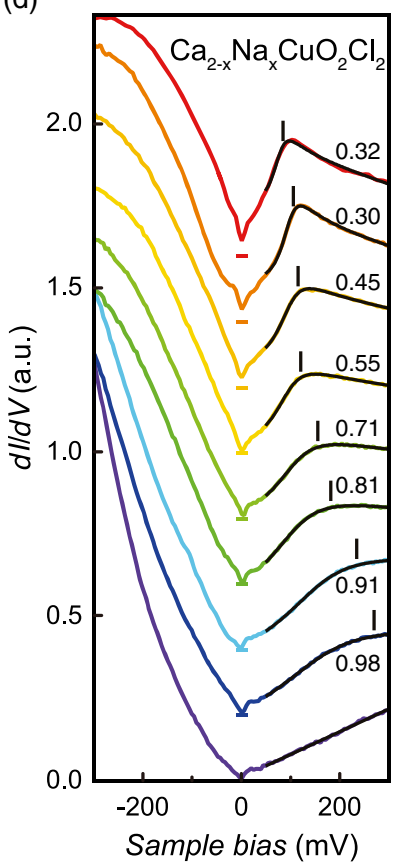

FIG. 5. (a) STM topography on the strongly insulating $x=0.30$ sample acquired with $V=100 \mathrm{mV}$ and $I=10 \mathrm{pA}$. (b) Typical $d I / d V$ curves (shifted vertically) taken at locations marked by the colored dots in (a), set up accordingly as Fig. 3(b) with $V=400 \mathrm{mV}$ and $I=180 \mathrm{pA}$. (c) Same as (b) but zoom-in on the low-energy range near $E_{F}$, which reveals a V-shaped gap. (d) $d I / d V$ spectra taken at various locations in lightly hole-doped Mott insulator $\mathrm{Ca}_{2-x} \mathrm{Na}_{x} \mathrm{Cu}_{2} \mathrm{OCl}_{2}(0.06 \leq x \leq 0.12)$. Adapted by permission from Y. Kohsaka, Nat. Phys. 8, 534 (2012). Copyright 2012, Macmillan Publishers Ltd. 
(a)

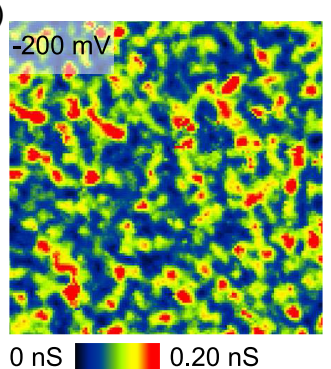

(c)

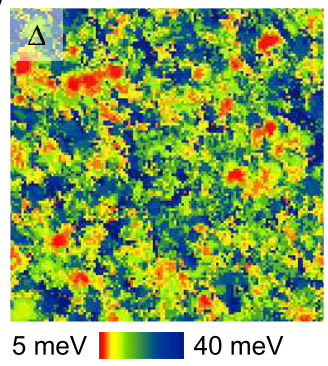

(b)

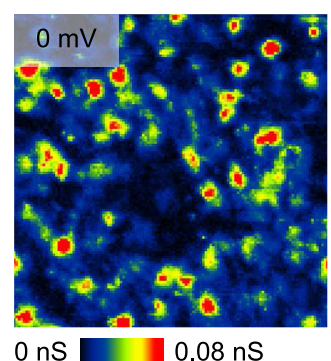

(d)

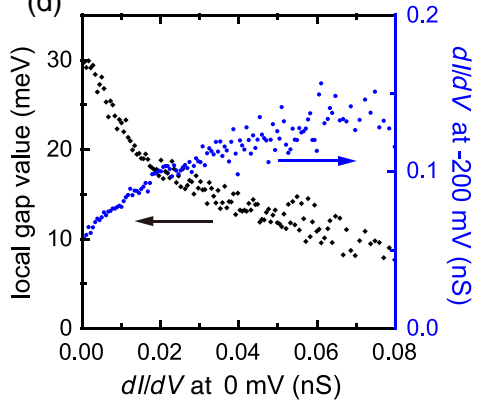

FIG. 6. (a),(b) $d I / d V$ image over the same field of view as in Fig. 5(a) with bias voltages of -200 and $0 \mathrm{mV}$. (c) Spatial distribution of the size of the V-shaped gap shown in Fig. 5(c). (d) Cross-correlation plot of the $-200 \mathrm{mV}$ peak height versus the local insulating gap magnitude (black) and residue DOS at $E_{F}$ (blue), revealing negative and positive correlations, respectively.

weight suppression has been observed in recent angleresolved photoemission spectroscopy (ARPES) measurements on $\mathrm{NaFe}_{1-x} \mathrm{Cu}_{x}$ As with $x$ up to 0.14 . Moreover, the core level spectrum reveals an increase of the high-energy electronic state at the binding energy of $-4 \mathrm{eV}$ [19]. Therefore, $\mathrm{Cu}$ substitution of $\mathrm{Fe}$ selectively reduces the spectral weight of the itinerant orbitals, and the extra electrons are injected into the localized orbitals. This is also consistent with the pronounced Curie-Weiss-type susceptibility due to enhanced local moments in the $x=0.30$ sample [Fig. 1(c)]. With reduced itinerant electron spectral weight and increased local moments, heavily $\mathrm{Cu}$-doped $\mathrm{NaFeAs}$ is close to the orbital-selected Mott transition [21-24]. The electron correlation effect is expected to be strongly enhanced in this regime [25].

We note that the majority of iron pnictides show weak correlation effect and remain metallic across the whole phase diagram. In this sense, the heavily $\mathrm{Cu}$-doped $\mathrm{NaFeAs}$ is an exception rather than the norm. The reason why this particular system possesses an insulating ground state can be understood from the two unique features of $\mathrm{Cu}$ substitution of Fe. The first is the systematic depletion of the itinerant electron spectral weight, which leads to the asymmetric line shape over the large energy range. In contrast, in Co-doped NaFeAs, the peak at $-200 \mathrm{mV}$ is nearly unaffected by $\mathrm{Co}$ [9]. Secondly, the $\mathrm{Cu}$ dopant induces a strong local impurity potential as manifested by the altered coherence peaks in the SC sample and pronounced inhomogeneity in the insulating sample. First-principles calculations

predicted that in the presence of substitutes with strong impurity potentials, the actual number of coherent carriers will diminish and localized states away from $E_{F}$ will emerge [26]. Both features are confirmed by our STM data and the ARPES results. The reduced itinerancy and strong disorderness can further enhance the correlation effect because the Coulomb repulsion cannot be efficiently screened under such circumstances [27]. The strong electron-electron interaction may cause a suppression of electron DOS at $E_{F}$, which naturally explains the small energy gap observed here in heavily $\mathrm{Cu}$-doped $\mathrm{NaFeAs}$. In contrast, the low-energy spectrum in heavily (11\%) Co-doped NaFeAs becomes rather featureless, which is characteristic of weakly interacting metal [9].

In summary, STM studies on $\mathrm{Cu}$-doped $\mathrm{NaFeAs}$ reveal a systematic evolution of the electronic structure from a superconductor to an insulator with spectral features strikingly similar to lightly doped cuprates. The insulating phase is most likely caused by the combined effect of reduced itinerant spectral weight and strong impurity potential due to $\mathrm{Cu}$ doping. These results suggest the relevance of the correlation effect in $\mathrm{Cu}$-doped $\mathrm{NaFeAs}$, which may serve as a link between the iron pnictides and cuprates. However, given the complexity associated with the $d I / d V$ spectra of both the cuprates and iron pnictides, we cannot exclude other explanations for the similarities reported here. Other experimental probes, especially resonant inelastic x-ray scattering spectroscopy, are needed to confirm whether the correlation effect plays an essential role in the electronic structure of this insulating iron pnictide.

\section{ACKNOWLEDGMENTS}

We thank Z. Y. Weng, G. M. Zhang, and Z. Sun for helpful discussions. This work was supported by the National Natural Science Foundation and MOST of China (2011CBA00101, 2012CB922002, 2015CB921000). A. W. and X. C. acknowledge support from the "Strategic Priority Research Program" of Chinese Academy of Sciences (Grant No. XDB04040100).

[1] P. A. Lee, N. Nagaosa, and X. G. Wen, Doping a Mott Insulator: Physics of High-Temperature Superconductivity, Rev. Mod. Phys. 78, 17 (2006).

[2] G. R. Stewart, Superconductivity in Iron Compounds, Rev. Mod. Phys. 83, 1589 (2011).

[3] D. C. Johnston, The Puzzle of High Temperature Superconductivity in Layered Iron Pnictides and Chalcogenides, Adv. Phys. 59, 803 (2010).

[4] D. J. Singh and M. H. Du, Density Functional Study of $\mathrm{LaFeAsO}_{1-x} \mathrm{~F}_{x}$ : A Low Carrier Density Superconductor Near Itinerant Magnetism, Phys. Rev. Lett. 100, 237003 (2008).

[5] C. Cao, P. J. Hirschfeld, and H. P. Cheng, Proximity of Antiferromagnetism and Superconductivity in $\mathrm{LaFeAsO}_{1-x} \mathrm{~F}_{x}$ : 
Effective Hamiltonian from Ab Initio Studies, Phys. Rev. B 77, 220506 (2008).

[6] P. C. Canfield, S. L. Bud'ko, N. Ni, J. Q. Yan, and A. Kracher, Decoupling of the Superconducting and Magnetic/Structural Phase Transitions in Electron-Doped $\mathrm{BaFe}_{2} \mathrm{As}_{2}$, Phys. Rev. B 80, 060501 (2009).

[7] N. Ni, A. Thaler, J. Q. Yan, A. Kracher, E. Colombier, S. L. Bud'ko, P. C. Canfield, and S. T. Hannahs, Temperature versus Doping Phase Diagrams for $\mathrm{Ba}\left(\mathrm{Fe}_{1-x} \mathrm{TM}_{x}\right)_{2} \mathrm{As}_{2}$ $(\mathrm{TM}=\mathrm{Ni}, \mathrm{Cu}, \mathrm{Cu} / \mathrm{Co})$ Single Crystals, Phys. Rev. B 82, 024519 (2010).

[8] A. F. Wang, J. J. Lin, P. Cheng, G. J. Ye, F. Chen, J. Q. Ma, X. F. Lu, B. Lei, X. G. Luo, and X. H. Chen, Phase Diagram and Physical Properties of $\mathrm{NaFe}_{1-x} \mathrm{Cu}_{x}$ As Single Crystals, Phys. Rev. B 88, 094516 (2013).

[9] X. Zhou, P. Cai, A. Wang, W. Ruan, C. Ye, X. Chen, Y. You, Z.-Y. Weng, and Y. Wang, Evolution from Unconventional Spin Density Wave to Superconductivity and a Pseudogaplike Phase in $\mathrm{NaFe}_{1-x} \mathrm{Co}_{x}$ As, Phys. Rev. Lett. 109, 037002 (2012).

[10] A. F. Wang, X. G. Luo, Y. J. Yan, J. J. Ying, Z. J. Xiang, G. J. Ye, P. Cheng, Z. Y. Li, W. J. Hu, and X. H. Chen, Phase Diagram and Calorimetric Properties of $\mathrm{NaFe}_{1-x} \mathrm{Co}_{x} \mathrm{As}$, Phys. Rev. B 85, 224521 (2012).

[11] G. M. Zhang, Y. H. Su, Z. Y. Lu, Z. Y. Weng, D. H. Lee, and T. Xiang, Universal Linear-Temperature Dependence of Static Magnetic Susceptibility in Iron Pnictides, Europhys. Lett. 86, 37006 (2009).

[12] H. Yang, Z. Wang, D. Fang, Q. Deng, Q.-H. Wang, Y.-Y. Xiang, Y. Yang, and H.-H. Wen, In-Gap Quasiparticle Excitations Induced by Non-Magnetic Cu Impurities in $\mathrm{Na}\left(\mathrm{Fe}_{0.96} \mathrm{Co}_{0.03} \mathrm{Cu}_{0.01}\right)$ As Revealed by Scanning Tunnelling Spectroscopy, Nat. Commun. 4, 2749 (2013).

[13] Y. Kohsaka, T. Hanaguri, M. Azuma, M. Takano, J. C. Davis, and H. Takagi, Visualization of the Emergence of the Pseudogap State and the Evolution to Superconductivity in a Lightly Hole-Doped Mott Insulator, Nat. Phys. 8, 534 (2012).

[14] L. P. Gor'kov and G. B. Teitel'baum, Dual Role of $d$ Electrons in Iron Pnictides, Phys. Rev. B 87, 024504 (2013).

[15] S. P. Kou, T. Li, and Z. Y. Weng, Coexistence of Itinerant Electrons and Local Moments in Iron-Based Superconductors, Europhys. Lett. 88, 17010 (2009).
[16] W. Lv, F. Krüger, and P. Phillips, Orbital Ordering and Unfrustrated $(\pi, 0)$ Magnetism from Degenerate Double Exchange in the Iron Pnictides, Phys. Rev. B 82, 045125 (2010).

[17] Y. J. Yan, P. Cheng, J. J. Ying, X. G. Luo, F. Chen, H. Y. Zou, A. F. Wang, G. J. Ye, Z. J. Xiang, J. Q. Ma et al., Structural, Magnetic, and Electronic Transport Properties of Hole-Doped $\mathrm{SrFe}_{2-x} \mathrm{Cu}_{x}$ As2 Single Crystals, Phys. Rev. B 87, 075105 (2013).

[18] M. Merz, P. Schweiss, P. Nagel, T. Wolf, H. v. Loehneysen, and S. Schuppler, To Dope or Not to Dope: Electronic Structure of Ba-Site and Fe-Site Substituted SingleCrystalline $\mathrm{BaFe}_{2} \mathrm{As}_{2}$, arXiv:1306.4222.

[19] S. T. Cui, S. Kong, S. L. Ju, P. Wu, A. F. Wang, X. G. Luo, X. H. Chen, G. B. Zhang, and Z. Sun, ARPES Study of the Effect of $\mathrm{Cu}$ Substitution on the Electronic Structure of NaFeAs, Phys. Rev. B 88, 245112 (2013).

[20] S. Ideta, T. Yoshida, I. Nishi, A. Fujimori, Y. Kotani, K. Ono, Y. Nakashima, S. Yamaichi, T. Sasagawa, M. Nakajima et al., Dependence of Carrier Doping on the Impurity Potential in Transition-Metal-Substituted FeAs-Based Superconductors, Phys. Rev. Lett. 110, 107007 (2013).

[21] R. Yu and Q. Si, Mott Transition in Multiorbital Models for Iron Pnictides, Phys. Rev. B 84, 235115 (2011).

[22] L. de' Medici, S. R. Hassan, M. Capone, and X. Dai, Orbital-Selective Mott Transition out of Band Degeneracy Lifting, Phys. Rev. Lett. 102, 126401 (2009).

[23] R. Yu, J. X. Zhu, and Q. Si, Mott Transition in Modulated Lattices and Parent Insulator of $(\mathrm{K}, \mathrm{Tl})_{y} \mathrm{Fe}_{x} \mathrm{Se}_{2}$ Superconductors, Phys. Rev. Lett. 106, 186401 (2011).

[24] M. Yi, D. H. Lu, R. Yu, S. C. Riggs, J. H. Chu, B. Lv, Z. K. Liu, M. Lu, Y. T. Cui, M. Hashimoto, et al., Observation of Temperature-Induced Crossover to an Orbital-Selective Mott Phase in $\mathrm{A}_{x} \mathrm{Fe}_{2-y} \mathrm{Se}_{2}(A=K, R b)$ Superconductors, Phys. Rev. Lett. 110, 067003 (2013).

[25] Q. Si and E. Abrahams, Strong Correlations and Magnetic Frustration in the High $T_{C}$ Iron Pnictides, Phys. Rev. Lett. 101, 076401 (2008).

[26] T. Berlijn, C.-H. Lin, W. Garber, and W. Ku, Do TransitionMetal Substitutions Dope Carriers in Iron-Based Superconductors?, Phys. Rev. Lett. 108, 207003 (2012).

[27] B. L. Altshuler and A. G. Aronov, Zero Bias Anomaly in Tunnel Resistance and Electron-Electron Interaction, Solid State Commun. 30, 115 (1979). 